\title{
DETERMINAÇÃO DOS COEFICIENTES DE DIFUSÃO TURBULENTA PARA A REGIÃO DO PORTO DE RIO GRANDE, RS, BRASIL
}

\author{
FONTOURA, J.A.S ${ }^{1}$; NIENCHESKI, L.F.H. ${ }^{2} \&$ ANTIQUEIRA, J.A. ${ }^{3}$ \\ 'Departamento de Materiais e Construção/FURG (fontoura@dmc.furg.br) \\ ${ }^{2}$ Laboratório de Hidroquímica/Departamento de Química/FURG \\ ${ }^{3}$ Laboratório de Oceanografia Geológica/Departamento de Geociências/FURG
}

\begin{abstract}
Fontoura, J.A.S.; Niencheski, L.F.H. \& Antiqueira, J.A. 2005. Determination of eddy diffusion coefficients for the area of Rio Grande harbor, RS, Brazil. Braz. J. Aquat. Sci. Technol. 9(2):87-92. ISSN 1808-7035. This work presents a study on the horizontal turbulent eddy diffusion for both inner part and shallow areas around of Rio Grande Harbor navigation channel, Patos Lagoon estuary, RS, Brazil. It was made through the monitoring of Rodamine 'B' dye using aerial photos and water samples. From the radiometric interpretation of aerial photos in concomitance with tracer concentration level measured on environment was determined two different diffusion modes, one into the navigation channel dominated by strong currents and other on shallow areas in agree with weak local hydrodynamics. The mean horizontal turbulent eddy diffusion (K) determined for whole event was about $10065 \mathrm{~cm}^{2} / \mathrm{s}$. Into the channel was identified a crescent diffusion rate with relation to the diffusion time and advection length, meanwhile in shallow areas was identified one decelerated process. The same was observed to the along flow eddy diffusion $(\mathrm{Kx})$ and cross flow eddy diffusion $(\mathrm{Ky})$. It's also verified which the longitudinal eddy diffusion $(\mathrm{Kx})$ varied from two to three times higher than transversal eddy diffusion (Ky) all process long. In shallow areas was also identified a eceleration on ratio between $\mathrm{Kx}$ and $\mathrm{Ky}$. This behavior suggests one more important dispersion process into the channel when compared with the marginal areas.
\end{abstract}

Keywords: Eddy diffusion, Fluorescent tracers, Dispersion of pollutants, Rodamine 'B'.

\section{INTRODUÇÃO}

O Porto do Rio Grande situa-se no estuário da Lagoa dos Patos, RS, no extremo sul do Brasil (Fig. 1). Nesta região a população concentra-se em duas cidades (Rio Grande e Pelotas) separadas por $50 \mathrm{Km}$ e responsáveis pelo lançamento de dejetos urbanos 'in natura' oriundos de esgotos cloacais não tratados. A ação antrópica pode ser subdividida em seis segmentos: complexo industrial portuário; conglomerado urbano; navegação interior e exterior; atividade primária; atividade pesqueira e turismo. De todos, apresentam-se como principais poluidores os quatro primeiros, respondendo pela maior parcela o complexo industrial portuário e os dejetos urbanos não tratados.

Essas atividades são responsáveis pelos elevados níveis de fosfato encontrados na enseada do Saco da Mangueira, principalmente junto às margens, provavelmente devido a emissões das indústrias de fertilizantes (Baumgarten et ali., 1995 e Baumgarten \& Niencheski, 1998), assim como, fortes evidências da presença de metais pesados oriundos de aporte antrópico (Baumgarten, 1987; Baumgarten et ali., 1990 e Baungarten \& Niencheski, op.sit.). Da mesma forma, foi constatada, a contaminação das águas de superfície por óleos e graxas, com ênfase especial para a área do Porto Novo e foz do estuário. A presença destes contaminantes é reflexo direto das atividades liga- das a navegação, como lavagem de porões, abastecimento de navios e vazamentos generalizados (Niencheski \& Baumgarten, 1997; Baumgarten \&Niencheski, op.sit.).

\section{MATERIAL E MÉTODOS}

A metodologia consistiu em gerar manchas superficiais com traçador fluorescente (Rodamina B) e monitorar sua dispersão com o auxílio de fotografias aéreas e amostragem seqüencial da água, em transectos ortogonais sobre as manchas (Fig. 2). Foram realizados cinco cruzeiros (1997 e 1998) para liberação do traçador e coleta de dados. Para realização dos trabalhos de campo foi utilizada a lancha oceânica Laruz (FURG) apoiada por um barco de menor porte para operação em águas rasas. A plataforma de vôo foi um Cessna 182 'Skylane', cedido pelo Aeroclube Cidade do Rio Grande, enquanto que as fotos foram realizadas com o auxilio do equipamento ADAR-1000, adquirido da Positive System Company, USA (Fontoura \& Hartmann, 2001).

As curvas contínuas de concentração de 'Rodamina B' foram obtidas através da correlação entre as absorbâncias determinadas nas amostras de água e as radiâncias extraídas das aerofotos, para pontos comuns (Fig. 3 e 4). Os valores dos coeficientes de 
difusão turbulenta horizontal $(K)$, longitudinal $(K x)$ e transversal (Ky) foram obtidos do acompanhamento da variação da área da mancha difusiva ao longo do tempo (Stevenson \& Inostroza, 1984). Os dados foram analisados com o auxilio dos programas ErMapper $5.2 \mathrm{e}$ Autocad R12. As correlações do coeficiente de difusão horizontal com o tempo de difusão, distância de advecção e coeficientes parciais foram obtidas por análise de regressão linear. Mais detalhes podem ser encontrados em Fontoura (2000).

\section{RESULTADOS E DISCUSSÃO}

As figuras 5, 6 e 7 apresentam o conjunto de curvas que descrevem o comportamento do coeficiente de difusão turbulenta horizontal (K) em função do tempo de difusão $(t)$, distância de advecção $(L)$ e velocidade da corrente na superfície (V). As curvas relacionando o coeficiente horizontal $(K)$ com o coeficiente longitudinal (Kx) e o transversal (Ky), são apresentadas nas figuras 8 e 9 .

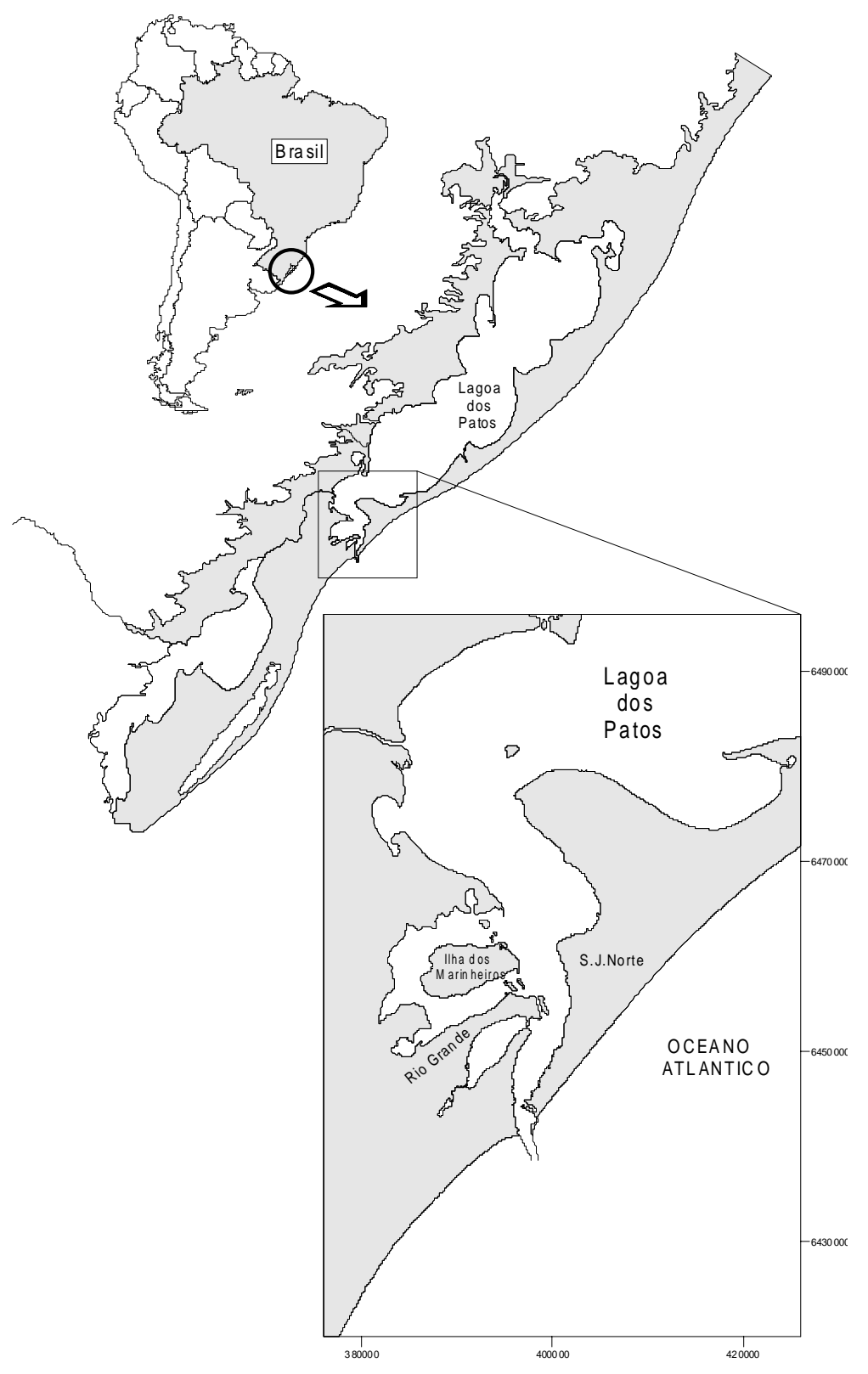

Figura 1 - Localização da área de estudo. 
De acordo com as figuras 5 e 6 observa-se que $o$ coeficiente $(\mathrm{K})$ apresentou comportamento diferenciado durante o processo, podendo ser identificadas duas etapas distintas: uma durante o período em que o traçador deslocou-se dentro da calha do canal e outra quando a mancha difusiva cruzou a borda do canal e começou a deslocar-se sobre a área rasa em direção a margem.

A primeira etapa contemplou aproximadamente $1 / 3$ do experimento e apresentou os maiores valores do coeficiente de difusão. Nesta fase a mancha percorreu a distância de $648 \mathrm{~m}$ a uma velocidade média de $0,63 \mathrm{~m} / \mathrm{s}$ e a área da mancha expandiu-se de $195 \mathrm{~m}^{2}$ até $1958 \mathrm{~m}^{2} \mathrm{em} 866 \mathrm{~s}$. Durante este percurso a mancha difusiva deslocou-se exclusivamente dentro do canal de navegação. A segunda etapa correspondeu aos restantes $2 / 3$ do experimento e indicou um comportamento decrescente para $(K)$, chegando este no final do experimento a apresentar valores até cinco vezes menor que os encontrados na etapa inicial. A mancha percorreu uma distância de $384 \mathrm{~m}$ com uma velocidade média de $0,20 \mathrm{~m} / \mathrm{s}$ e a área da mancha cresceu de $1958 \mathrm{~m}^{2}$ até $2879 \mathrm{~m}^{2} \mathrm{em} 1896 \mathrm{~s}$. Durante esta fase a mancha cruzou a borda do canal em direção à zona rasa adjacente e deslocou-se sobre ela em direção a margem. O comportamento advectivo da mancha difusiva pode ser observado na figura 2.

A figura 7 mostra a relação existente entre o coeficiente de difusão $(K)$ e a velocidade da corrente na superfície (V). A adoção da velocidade da corrente na superfície como parâmetro de comparação só foi possível graças às condições meteorológicas do período,

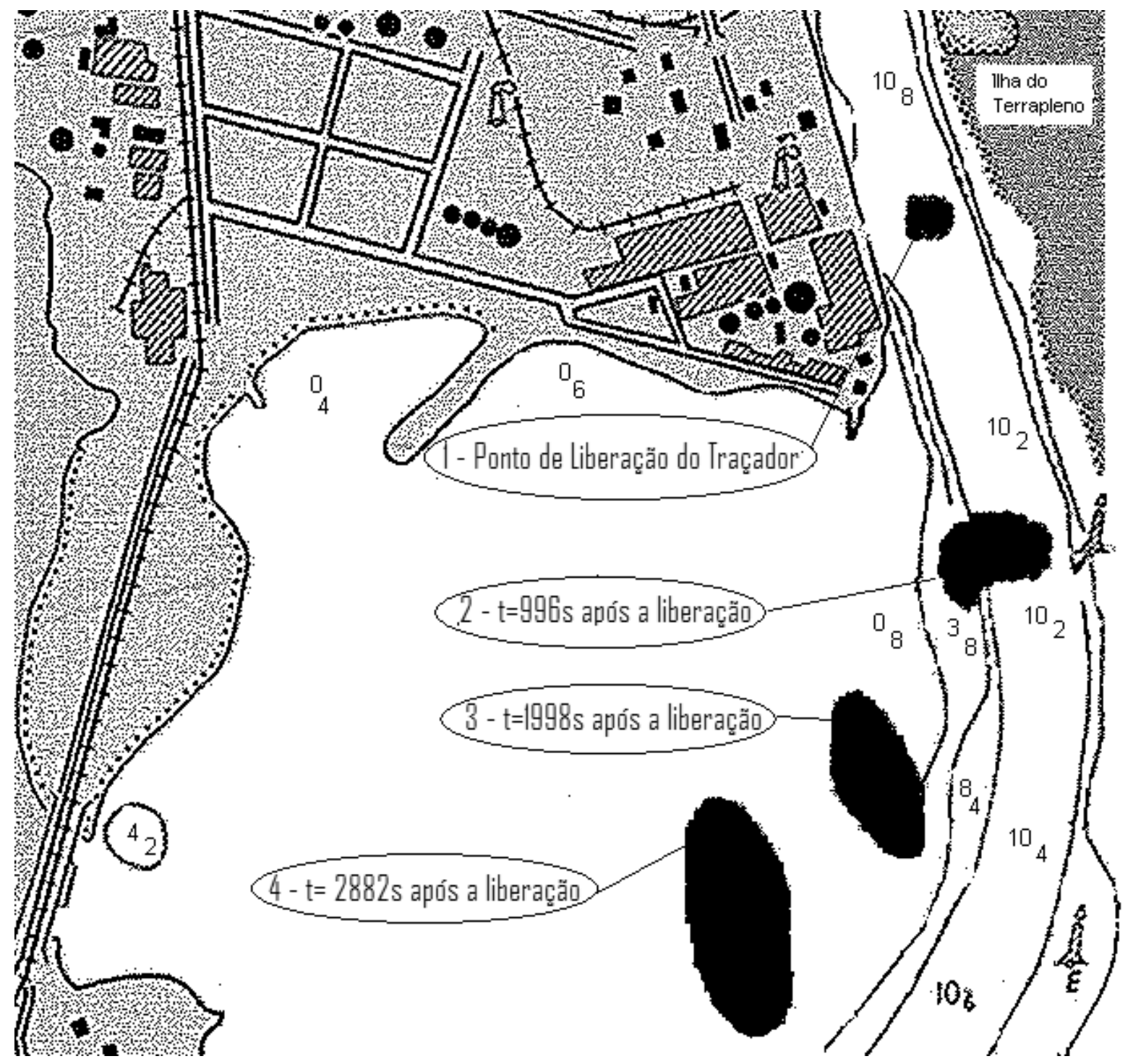

Figura 2 - Localização do experimento de 07/08/98. 


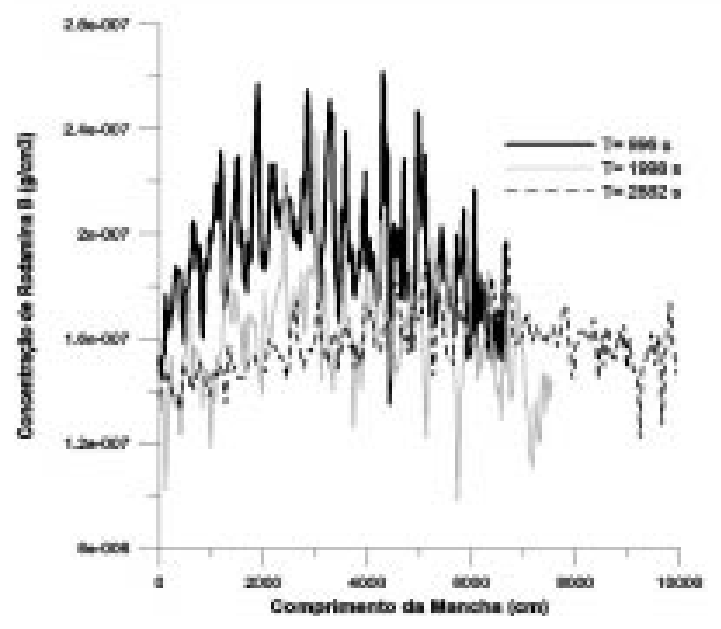

Figura 3 - Curvas de distribuição das concentrações de Rodamina $B$ ao longo do eixo longitudinal da mancha em momentos sucessivos do evento.

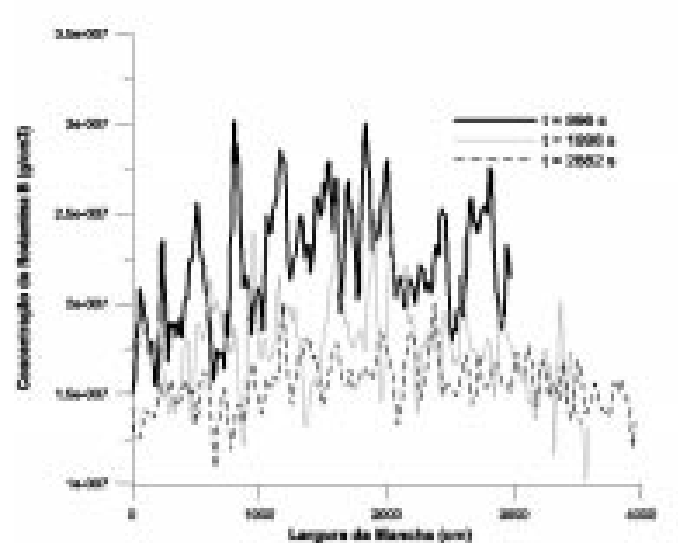

Figura 4 - Curvas de distribuição das concentrações de Rodamina $B$ ao longo do eixo transversal da mancha em momentos sucessivos do evento.

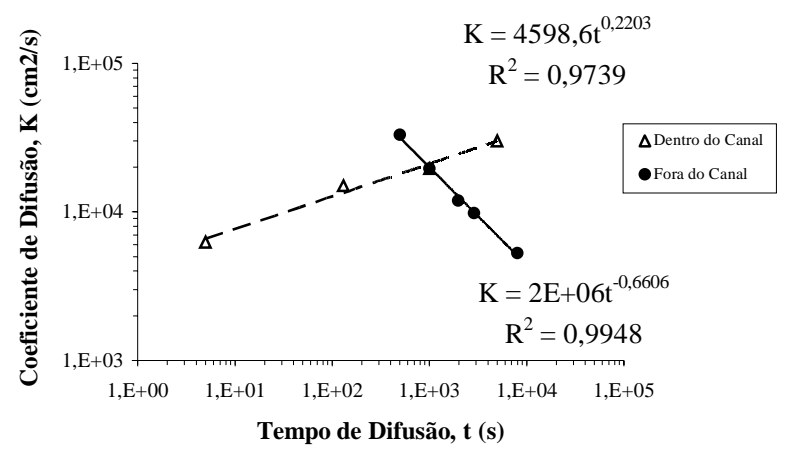

Figura 5 - Relação entre o coeficiente de difusão turbulenta e o tempo de difusão. que geraram grandes descargas continentais, capazes de impedir correntes de fundo oriundas da intrusão salina, que em conjunto com a profundidade rasa do estuário, permitiu assumir um perfil vertical homogêneo de velocidades no período estudado. Os valores de (K) variam em função da velocidade média na superfície. Dentro do canal de navegação o crescimento rápido do coeficiente de difusão está associado aos valores mais altos da corrente $(0,63 \mathrm{~m} / \mathrm{s})$, na medida que águas mais rasas são atingidas os valores de $(\mathrm{K})$ decrescem junto com a velocidade média $(0,10 \mathrm{~m} / \mathrm{s})$. Isto explica as concentrações de nutrientes encontradas por Niencheski \& Windom (1994) no centro do canal de navegação e consideradas normais para estuários não contaminados, em situação nitidamente contrária aos valores encontrados por Baungarten et ali. (1995) e Baungarten et ali. (em preparo) para águas marginais. Observando a figura 2 verificamos que a mancha difusiva apresenta forma assimétrica, ou seja, o eixo longitudinal à direção principal do fluxo cresce mais rapidamente que o eixo transversal ao mesmo. Isto significa que a difusão é maior nesta direção. Este comportamento assimétrico pode ser descrito pela inserção dos conceitos de coeficiente de difusão longitudinal $(\mathrm{Kx})$ e coeficiente de difusão transversal (Ky). As figuras 8 e 9 mostram a relação existem entre o coeficiente de difusão $(K)$ e os coeficientes (Kx) e (Ky). Observa-se novamente o surgimento de fases distintas com (Ky) mantendo-se aproximadamente estável durante o evento, enquanto que $(\mathrm{Kx})$ flutua entre uma maior taxa de crescimento dentro do canal de navegação e uma desaceleração nas áreas marginais. Embora este último tenha se mantido duas a três vezes maior que o primeiro durante todo o evento.

\section{CONCLUSÃO}

A metodologia empregada mostrou-se adequada à obtenção dos coeficientes de difusão turbulenta horizontal (K), longitudinal (Kx) e transversal (Ky) para o canal de navegação e rasos adjacentes na área do porto do Rio Grande. A qualidade, confiabilidade e abrangência dos coeficientes é limitada pelo número de experimentos realizados e diversidade das situações hidrodinâmicas encontradas. O coeficiente de difusão turbulenta horizontal $(\mathrm{K})$ considerando o conjunto dos dois ambientes, apresentou um valor médio na ordem de $10065 \mathrm{~cm}^{2} / \mathrm{s}$, entretanto valores médios parecem não ser o melhor caminho a se adotar para trabalhos na região, pois as diferenças hidrodinâmicas entre os dois ambientes são muito acentuadas e podem comprometer as interpretações baseadas em valores comuns. Embora os valores encontrados descrevam o comportamento difusivo dos ambientes estudados su- 


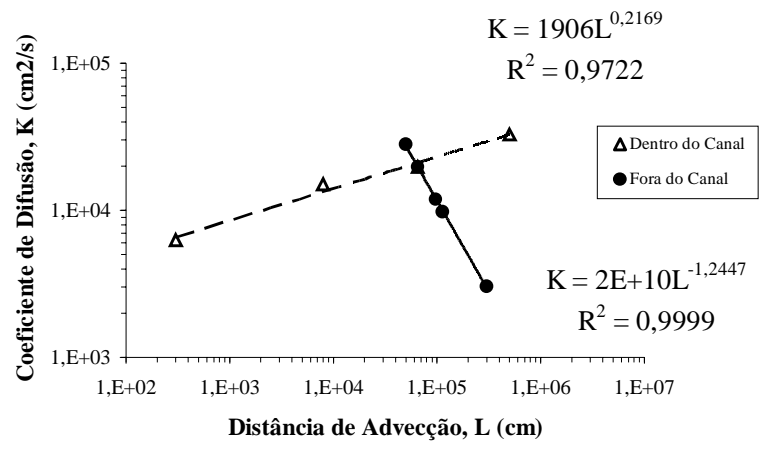

Figura 6 - Relação entre o coeficiente de difusão turbulenta e a distância de advecção.

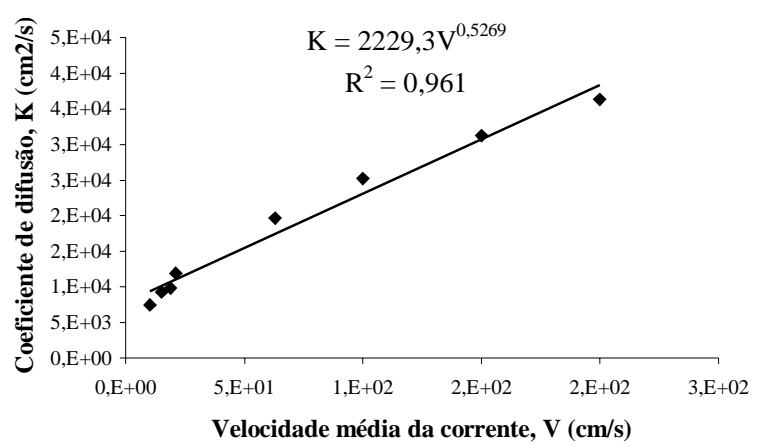

Figura 7 - Relação entre o coeficiente de difusão turbulenta e a velocidade média da corrente na superfície.

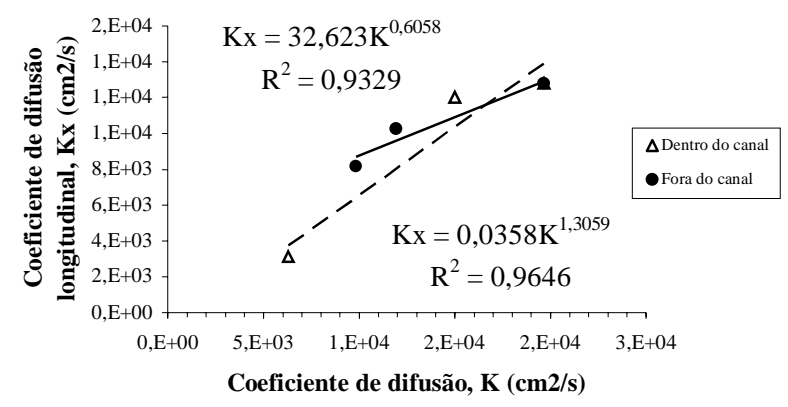

Figura 8 - Relação entre o coeficiente de difusão turbulenta horizontal e o coeficiente de difusão turbulenta longitudinal.

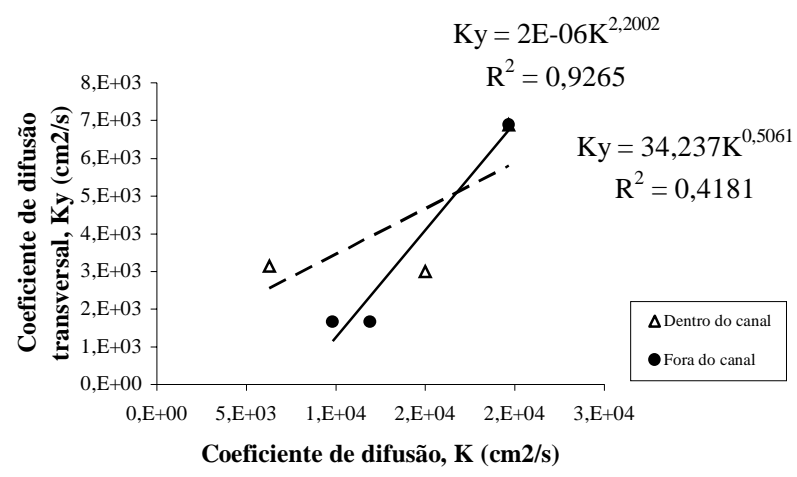

Figura 9 - Relação entre o coeficiente de difusão turbulenta horizontal e o coeficiente de difusão turbulenta transversal. gere-se o prosseguimento das investigações através de um maior número de experimentos que contemplem diferentes pontos do estuário e condições hidrodinâmicas variadas.

\section{REFERÊNCIAS}

Baumgarten, M.G.Z. 1987. Avaliação do Balanus Improvisus como indicador dos níveis metálicos do estuário da lagoa dos Patos (RS, Brasil). Dissertação de mestrado. FURG. 178 p.

Baumgarten, M.G. \& Niencheski, L.F. 1998. Avaliação da qualidade hidroquímica da área portuária

da cidade do Rio Grande. Série Documentos Técnicos № 9 / Oceanografia - FURG. Editora da FURG. 66p.

Baumgarten, M.G.Z.; Klein, A.H.F. \& Niencheski, L.F.H. 1990. Níveis de cobre, zinco e chumbo dissolvidos na lagoa dos Patos (RS). IN: Anais do II simpósio de ecossistemas da costa sul e sudeste brasileira: estruturação, função e manejo. 6 a 11 de abril. Águas de Lindóia (SP): Vol. II, p. 117-126.

Baumgarten, M.G.Z.; Niencheski, L.F.H. \& Kuroshima, K.N. 1995. Qualidade das águas estuarinas que margeiam o município do Rio Grande (RS): nutrientes e detergentes dissolvidos. Revista Atlântica. Vol. 17: 17-34

Baumgarten, M.G.Z.; Veeck, L. \& Niencheski, L.F.H. (em preparo). Nutrientes na água intersticial de uma enseada rasa do estuário da lagoa dos Patos. Resumo. Congresso de iniciação científica - prêmio jovem cientista. FURG-UFPEL-UFSM. Pelotas.

Bordas, M.P.; Casalas, A.; Silveira, A. \& Gonçalves, M. 1984. Circulação e dispersão em sistemas costeiros e oceânicos. Caso da lagoa dos Patos. Tech. Report IPH/UFRGS. Brazil.

Fontoura, J.A.S. 2000. Uso da 'Rodamina B' na validação de um modelo numérico de dispersão em superfície, no estuário da Lagoa dos Patos. Dissertação de mestrado. Engenharia Oceânica/FURG. Rio Grande, RS. 156 p.

Fontoura, J.A.S. \& Hartmann, C. 2001. Capture small format aerial digital images using the airborne data acquisition system (ADAR-1000) from Positive System Company (USA). Pesquisas em Geociências, 28 (2): 373-381, 2001.

Lam, D.C.L.; Murthy, C.R. \& Simpson, R.B. 1984. Lecture notes on coastal and estuarine studies. Effluent transport and diffusion models for the coastal zone. Springer-Werlag New York Inc. 168 p.

Möller Jr., O.O.; Paim, P.S. \& Soares, I. 1991.Facteurs et mecanismes de la circulacion des eaux dansl'estuarie de la lagune dos Patos. Bulletim Institute de Geologie du Bassin d'Aquitaine, 49 : 15-21. 
Möller Jr., O.O. 1996. Hidrodynamique de la lagune dos Patos $\left(30^{\circ} \mathrm{S}\right.$, Brésil). Mesures et modélisation. Tese Doutorado apresentada a Universidade de Bordeaux. França. $200 \mathrm{p}$.

Niencheski, L.F.H. \& Windom, H. 1994. Nutrient flux and budget in Patos lagoon estuary. The Science of the Total Environment. Vol. 149 (1-2): 53-60.

Niencheski, L.F.H. \& Baumgarten, M.G.Z. 1997. Projeto: Relatório de impacto do meio ambiente das atividades do porto de Rio Grande. Sub-Projeto: Avaliação da qualidade hidroquímica da área portuária da cidade do Rio Grande. 45 p.
Rochefort, M. 1958. Rapports entre la pluviosité et l'écoulement dans le Brésil subtropical et le Brésil tropical Atlantique. Travaux et mémoires de l'Institut des hautes etudes de l'Amérique latine. $265 \mathrm{p}$.

Stevenson, M.R. \& Inostroza, H.M.V. 1984. Diffusion coefficients for coastal waters determined from aerial photographs. IN: Proceedings of IV annual meeting of selper (sociedad de especialistas latino americanos en percepción remota). Santiago. Chile. November 12-18.

Submetido: Outubro/2004

Revisado: Fevereiro/2005 Aceito: Setembro/2005 